\title{
Pelvic ateriovenous malformation leading to repeated episodes of haematuria in an adult with history of paralytic poliomyelitis: a case report
}

\begin{abstract}
Background: Pelvic arteriovenous malformations are rare and can be either congenital or acquired in origin. Pelvic arteriovenous malformations, when large, can present with number of symptoms such as pelvic pain, haematuria, dysuria, urinary retention, impotence and rectal tenesmus. Proper imaging can guide the treatment of these lesions and hence prevent the complications associated with them.

Case presentation: We present a case of 39 year old male with the chief complaint of pelvic discomfort and on and off haematuria. It was not associated fever or preceding trauma. Patent had history of childhood paralytic poliomyelitis. Because of the deranged renal function, MR evaluation of pelvis was done which demonstrated arteriovenous malformation abutting the right lateral urinary bladder wall, seminal vesicle and prostate.

Conclusion: Though pelvic vascular malformations are rare, they when occur, can lead to repeated vague pelvic symptoms. Proper imaging with early diagnosis and treatment can help in significantly reducing the mortality and morbidity associated with these lesions.
\end{abstract}

Volume 10 Issue 5 - 2020

\author{
Nirmal Prasad Neupane,' Keshika Koirala ${ }^{2}$ \\ 'Department of Radiology, Grande International Hospital, Nepal \\ ${ }^{2}$ Department of Radiology, Nepal Medical College Teaching \\ Hospital, Nepal
}

\begin{abstract}
Correspondence: Nirmal Prasad Neupane, Radiologist Grande International Hospital Kathmandu, Nepal Email nirmal.nup@gmail.com
\end{abstract}

Received: September 02, 2020 | Published: October 28, 2020

Keywords: case report, pelvic arteriovenous malformation, haematuria, urinary bladder wall thickening, paralytic poliomyelitis

\section{Background}

Arteriovenous malformation refers to a high flow vascular lesion and is formed by multiple abnormal communications between the arterial and venous systems. Pelvic arteriovenous malformations are rare and can be either congenital or acquired in origin. ${ }^{1}$ In acquired cases, they usually occur secondary to neoplasms, pelvic trauma, or pelvic surgical procedures. ${ }^{1,2}$ Pelvic arteriovenous malformations, when large, can present with a number of symptoms such as pelvic pain, haematuria, dysuria, urinary retention, impotence and rectal tenesmus. We present a case of congenital pelvic arteriovenous malformation who had repeated episodes of haematuria. Since the patient had deranged renal function tests, we evaluated him with MR study of the pelvis.

\section{Case report}

A 39 year old male presented with the chief complaint of pelvic discomfort and on and off haematuria. It was not associated with fever or urinary retention. No bowel symptoms were noted. Patient had history of paralytic poliomyelitis in his childhood and walked with crutches. On laboratory examination, patient was found to have deranged renal function tests with elevated creatinine level. Hence, we conducted MRI pelvis with MR urography to look for the urinary systemand to look for the status of the the pelvic muscles and joints. MR study showed multiple tortuous dilated vessels on the right side of pelvis suggestive of pelvic arteriovenous malformation (Figure $1 \& 2)$. The arterial supply was noted from multiple branches of the right internal iliac artery and venous drainage was noted into right external iliac vein and deep external pudendal vein. The tangle of vessels was abutting the right lateral wall of urinary bladder, prostate and right seminal vesicle medially (Figure $1 \& 3$ ). It was abutting the obturatorinternus muscle laterally. There wasasymmetric thickening of the right lateral wall of urinary bladder adjacent to the vascular malformation (Figure 1). It was not associated with perivesicular fat strandings or enlarged locoregional lymphnodes. No restriction of diffusion was noted in DWI images. Prostate and bilateral bilateral seminal vesicles were normal in signal intensity and morphology. As a sequalae of childhood poliomyelitis, diffuse atrophy of pelvic muscles was noted that was more prominent on the right side (Figure 4\&5). T1 and T2 high signal intensity suggestive of fatty replacement was noted in bilateral psoas and right obturaterinternus mucle. Fatty atrophy of bilateral hip and proximal thigh muscles were also seen (Figure 5). Abduction and external rotation was noted at bilateral hip joints (Figure 4). Histological examination of the bladder wall revealed polypoid bladder mucosa with chronic inflammation consistent with polypoid cystitis. Arteriovenous malformation was managed with intra-arterial coil embolization and subsequently the patient's symptoms improved.

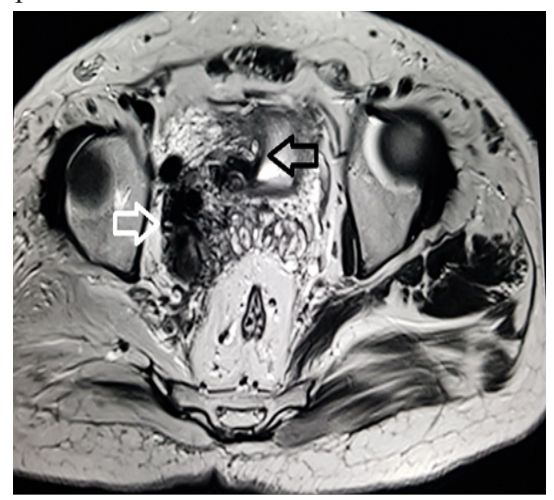

Figure I MRI axial T2 weighted image through the pelvis demonstrate multiple tortuous vessels (white arrow) on the right side extending to the right lateral wall of urinary bladder. The vessels are dilated and show loss of signal suggestive of high flow vascular malformation.Associated mild thickening of right lateral wall of urinary bladder is also noted (black arrow). 


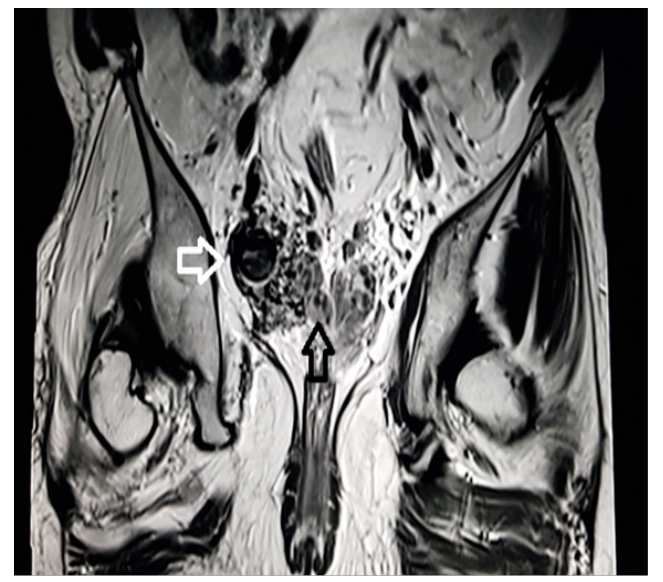

Figure 2 MRI coronal T2 weighted image through the pelvis demonstrate dilated tortuous vessels (white arrow) on the right side of pelvis abutting the right seminal vesicle (black arrow).

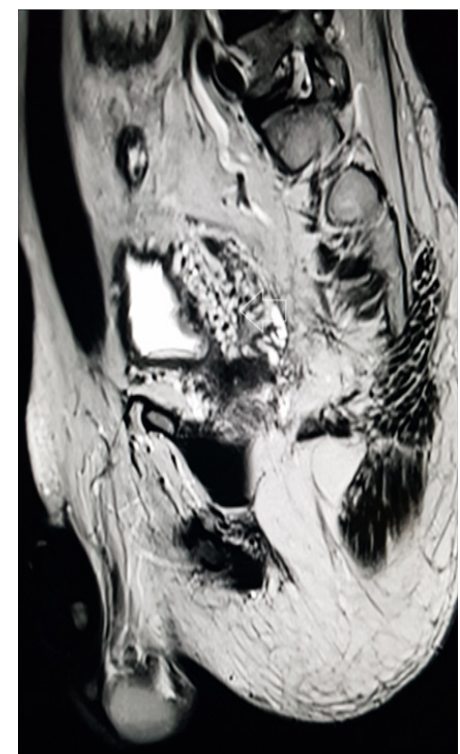

Figure $3 \mathrm{MRI}$ sagittalT2 weighted image through the pelvis demonstrate multiple tangle of vessels (white arrow) on the right posterolateral aspect of urinary bladder.

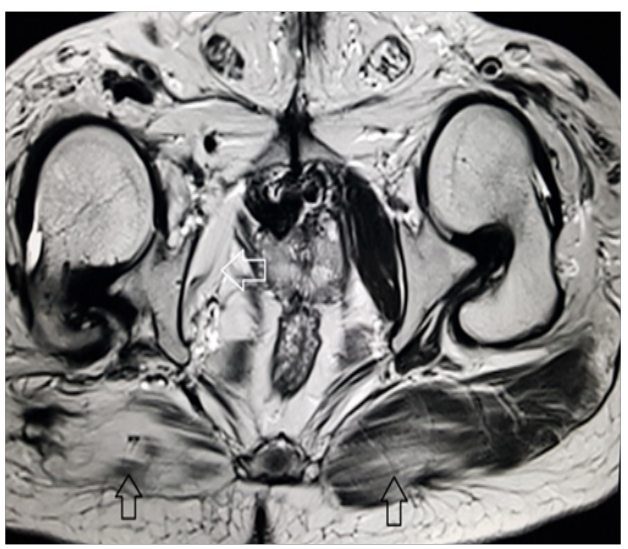

Figure $4 \mathrm{MRI}$ axial T2 weighted image through the lower pelvis demonstrate diffuse fatty atrophy of the gluteal muscles (black arrows) that is more prominent on the right side. Marked fatty atrophy of right obturatorinternus muscle is also noted (white arrow). External rotation of bilateral hip joint is demonstrated. These findings represent sequalae of childhood paralytic poliomyelitis.

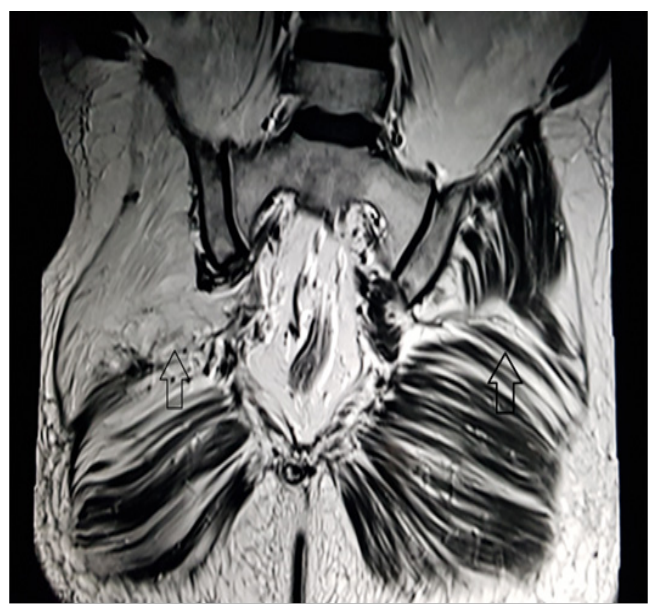

Figure $5 \mathrm{MRI}$ coronal $\mathrm{TI}$ weighted image through the gluteal region demonstrate diffuse fatty atrophy of the gluteal muscles (black arrows) that is more prominent on the right side. It occurred as a sequalae of paralytic poliomyelitis.

\section{Discussion}

\section{Introduction and general epidemiology}

Arteriovenous malformations are high flow vascular malformations that consist of feeding arteries, draining veins, and a nidus. The nidus is composed of multiple dysplastic vascular channels that connect the arteries and veins. No intervening capillary networks are present in these malformations. ${ }^{3}$ Pelvic arteriovenousmal formations are rare in occurrence and are twice as common in women as in men..$^{1,2}$ They can be either congenital or acquired in origin. In women, they are typically related to previous gynecologic surgeries, prior childbirth and gestational trophoblastic diseases. They can also occur secondary to trauma and pelvic malignancies. Patients with these malformations may have a number of nonspecific symptoms including pain, haematuria, dyspareunia, impotence, and changes in bowel and bladder function.

\section{Imaging findings}

Various imaging modalities can be used for the evaluation of the vascular malformations. Traditionally, ultrasound has been the imaging modality of choice for initial assessment and characterization of these vascular lesions. ${ }^{4}$ However, ultrasonogram has its limitations in the form of narrow field of view, restricted three-dimensional (3D) imaging, and operator dependency. ${ }^{5}$ Magnetic resonance imaging (MRI) has now become the most valuable modality for classification of vascular anomalies. ${ }^{6}$ It accurately demonstrates the extension of the lesion and its anatomic relationship to adjacent structures.

Ultrasonography with Doppler study, in these lesions, shows erpiginoustubular anechoic structures with low resistance and highvelocity flow pattern. It can be useful in differentiating the vascular lesion from anechoic collections and other pelvic cystic lesions. Contrast enhanced CT and MRI, both are non-invasive modalities and are helpful to demonstrate vascular etiology of the lesion and to evaluate its extension. These modalities can also demonstrate the feeding arteries, draining veins and the involvement of adjacent structures. Conventional angiography, on the other hand, is usually carried out before therapeutic intervention of these lesions. It can serve diagnostic as well as therapeutic purpose in the same setting as it can be used to guide the embolization of the vessels. 


\section{Management and prognosis}

Small asymptomatic pelvic arteriovenous malformations can be followed up conservatively. However, larger ones need surgical or endovascular treatment. ${ }^{1,7}$ Surgical treatment consists of resection of the malformation and ligature of the feeding arteries. ${ }^{8}$ Arteriovenous malformations of the pelvis are often supplied by multiple arteries including the anterior branches of the internal iliac arteries, the inferior mesenteric arteries, and the median sacral artery. Because of the extensive vascular supply, surgical resection of these lesions is difficult and carries the risk of a massive intraoperative haemorrhage. Incomplete removal of thenidus, surrounding organ injury, and high recurrence rates are other complications associated with the surgical management of these lesions. ${ }^{8,9}$ Therefore, endovascular therapy with various embolic and sclerosing materials, independently or combined with surgical treatment, has become the therapeutic option of choice for these lesions. ${ }^{7,8}$ Various embolic materials such as rapidly polymerizing acrylic adhesives (e.g., n-butyl cyanoacrylate or isobutyl cyanoacrylate), polyvinyl alcohol foam particles, ethylenevinyl alcohol copolymers (Onyx) and endovascular coils can be used in the treatment of these vascular lesions (Table 1).

Table I Case summary

\begin{tabular}{lll}
\hline I & Patient (gender, age) & 39 year old male \\
2 & Final Diagnosis & Pelvic arteriovenous malformation \\
3 & Symptoms & Haematuria \\
4 & Medications & None \\
5 & Clinical Procedure & Intra-arterial coil embolization \\
6 & Specialty & Radiology \\
\hline
\end{tabular}

\section{Conclusion}

Though pelvic vascular malformations are rare, they when occur can lead to repeatedpelvic symptoms. Pelvic vascular malformations should be kept as a differential in these patients especially when associated with history of previous pelvic malignancies and surgeries. Proper imaging with early diagnosis and treatment can help in significantly reducing the mortality and morbidity associated with these lesions.

\section{Acknowledgments}

None.

\section{Funding}

None.

\section{Conflicts of interest}

The authors declare that they have no competing interests and no financial disclosures to be made.

\section{References}

1. Game X, Berlizot P, Hassan $T$, et al. Congenital pelvic arteriovenous malformation in male patients: a rare cause of urological symptoms and role of embolization. European urology. 2002;42(4):407-412.

2. Smith MR. Pulsative pelvic masses: Options for evaluation and management of pelvic arteriovenous malformations. American Journal of Obstetrics \& Gynecology. 1995;172(6):1857-1863.

3. Legiehn GM, Heran MK. Classification, diagnosis, and interventional radiologic management of vascular malformations. Orthopedic Clinics. 2006;37(3):435-474.

4. Dubois J, Alison M. Vascular anomalies: what a radiologist needs to know. Pediatr Radiol. 2010;40(6):895-905.

5. Moukaddam H, Pollak J, Haims AH. MRI characteristics and classification of peripheral vascular malformations and tumors. Skeletal Radiol. 2009;38(6):535-547.

6. Flors L, Leiva-Salinas C, Maged IM, et al. MR imaging of soft-tissue vascular malformations: diagnosis, classification, and therapy follow-up. Radiographics. 2011;31(5):1321-1341.

7. Jacobowitz GR, Rosen RJ, Rockman CB, et al. Transcatheter embolization of complex pelvic vascular malformations: results and long-term followup. J Vasc Surg. 2001;33(1):51-55.

8. Trout HH 3rd, McAllister HA Jr, Giordano JM, Rich NM. Vascular malformations. Surgery. 1985;97(1):36-41.

9. Pritchard DA, Maloney JD, Bernatz PE, et al. Surgical treatment of congenital pelvic arteriovenous malformation. Mayo Clin Proc. 1978;53(9):607-611. 